\title{
A Framework for Assessing Reading Comprehension of Geometric Construction Texts
}

\author{
Kai-Lin $\operatorname{Yang}^{1}$ (D) Jian-Lin $\mathbf{L i}^{2}$
}

Received: 10 December 2015 / Accepted: 22 August 2016 /Published online: 1 September 2016

(C) The Author(s) 2016. This article is published with open access at Springerlink.com

\begin{abstract}
This study investigates one issue related to reading mathematical texts by presenting a two-dimensional framework for assessing reading comprehension of geometric construction texts. The two dimensions of the framework were formulated by modifying categories of reading literacy and drawing on key elements of geometric construction texts. Three categories of reading mathematical texts were recognized and then cross-tabulated with three key elements of geometric construction texts to create a nine-category assessment framework, which was used to design an instrument. After reporting on the validation of the instrument, we conclude by discussing the implications of the framework for assessing students' reading to learn mathematics and for improving the learning of geometric constructions by reading.
\end{abstract}

Keywords Comprehension · Geometry construction $\cdot$ Reading $\cdot$ Text

\section{Introduction}

In view of the importance of assessing reading comprehension in developing students' ability to learn by reading, many researchers have investigated how to measure reading ability (e.g. Sabatini, Albro \& O'Reilly, 2012). However, good readers of general texts are not necessarily good readers of mathematics texts (Shepherd, Selden \& Selden, 2012). According to Fang and Schleppegrell (2010), "mathematical discourse is simultaneously technical, dense, and multi-semiotic, drawing on natural language, symbolic language, and visual display, which interact in discipline-specific, synergistic ways" (p. 591). Whereas mathematics has its special syntax and semantics, mathematics

Kai-Lin Yang

kailin@ntnu.edu.tw

1 Department of Mathematics, National Taiwan Normal University, No. 88, Ting-Chou Rd. Sec. 4, Taipei, Taiwan

2 Guang Ming Junior High School, Taoyuan, Taiwan 
education researchers have revealed that students' reading comprehension of mathematics proof is complex and called for developing comprehensive frameworks for assessing students' ability to learn mathematics by reading (Conradie \& Frith, 2000; Mejia-Ramos, Fuller, Weber, Rhoads \& Samkoff, 2012; Selden \& Shepherd, 2013; Yang \& Lin, 2008). The aim of this paper is to conceptualize an assessment framework and to apply it to designing questions to investigate students' reading comprehension of geometric construction texts.

Duval (2006) pointed out three kinds of cognitive processes in understanding geometry: visual process; construction of figures; and discursive process for exploring, explaining, or proving. Construction draws on discursive process and leads to visual process. In order to justify what is constructed, not only natural discursive process, which embeds visual process, but also deductive discursive process are required. Thus, geometric construction can play a role to bridge the gap between visualization and deductive reasoning. For decades, researchers have contended that geometric constructions are useful in (1) lending visual clarity to many geometric relationships (Sanders, 1998), (2) making something tangible for secondary school students (Robertson, 1986), and (3) promoting a spirit of exploration and discovery (Pandiscio, 2002). All these ideas also suggest that the learning of geometric constructions has the potential to bridge the gap between visualization (visual process) and deductive reasoning (discursive process) in the sense of Duval (1995). For school geometry, it may be somehow arguable how much attention should be paid to teaching geometric constructions. Nonetheless, the content of constructing geometrical figures using compass and straightedge is still included in many national curricula, for example, in Australia, USA, and England (Australian Curriculum, Assessment and Reporting Authority, 2012; Common Core State Standards Initiative, 2012; Department for Education, 2013).

Schoenfeld (1986) found that most students who had studied 1-year high school geometry were "naive empiricists whose approach to straightedge-and-compass constructions is an empirical guess-and-test loop" (p. 243). Other studies have also shown that the learning of geometric constructions is important but not easy for students due to the need to distinguish between drawings and figures (e.g. Hölzl, 1995), as well as between spatial-graphical realities and geometrical relations (e.g. Laborde, 1998). Those studies uncovered students' difficulties in solving geometric construction tasks.

Most of the previous studies on the learning of geometric constructions are based on the perspective of learning by doing or the use of dynamic geometry software. Our concern is about the perspective of learning by reading that is portrayed as "a learning process inasmuch as the reader is said to transform the text in the act of reading" (Borasi \& Siegel, 1989, p.11). Thus, it is interesting to investigate how well students can read to understand mathematical texts. We selected the geometric construction texts, Euclidean constructions using compass and straightedge, for this study because its content includes both procedural/conceptual knowledge and mathematical reasoning (Pandiscio, 2002; Sanders, 1998; Schoenfeld, 1986). Herein, a geometric construction text includes three parts of information: (1) one construction task (or problem), (2) the construction steps used to solve the task, and (3) the figures corresponding to each construction step. On the one hand, conceptual and procedural knowledge are required to understand geometric construction steps. On the other hand, mathematical reasoning is required to understand the logical relations among construction steps and their corresponding figures. 
Figure 1 shows a geometric construction text on how to construct the perpendicular bisector of a given line segment and will be discussed theoretically as an example. When reading this text, students may not be able to comprehend step 1 if they do not know how to construct a circle with a compass. Even though the students know how to construct a circle with a compass, they still may not be able to properly apply the skill due to their misunderstanding of the interaction of two arcs constructed by a compass with the same radius as center (interview data). Moreover, they may not be able to recognize the property of a compass, that is, the distance between the two points of a compass is the same when they are fixed, and know why the two steps are needed for constructing the perpendicular bisector of a given line segment, that is, the two diagonals of a rhombus are perpendicular bisectors (Yang, 2015). To further understand the reading comprehension of geometric construction texts, we conceptualize a framework for this from the perspectives of reading literacy (Kirsch, de Jong, LaFontaine, McQueen, Mendelovits \& Monseur, 2002), geometric cognition (Duval, 1995), mathematical literacy (Organisation for Economic Cooperation and Development (OECD), 2004), and geometric constructions drawn from reading comprehension of geometric proof (Yang \& Lin, 2008).

\section{Conceptualized Reading Comprehension of Geometric Construction Texts}

For conceptualizing reading comprehension of geometry construction texts, the first dimension refers to reading literacy in the Program for International Student Assessment (PISA), which is proposed for the evaluation of students" "understanding, using and reflecting on written texts, in order to achieve one's goals, to develop one's knowledge and potential and to participate in society" (Kirsch et al., 2002, p. 25).

[Geometric Construction Task]

Given $\overline{A B}$, construct the perpendicular bisector of $\overline{A B}$.

$A \bullet \longrightarrow B$

[Step 1]

Take pints A and B as the centers of two circles, and for each point, draw an arc with a radius of the same length which is longer than half of the length of $\overline{A B}$. The two points where these two arcs intersect are called $\mathrm{C}$ and $\mathrm{D}$, respectively.

[Step 2]

Draw the line to link the two points, $C$ and D. Line $\mathrm{CD}$ is the perpendicular bisector of $\overline{A B}$.
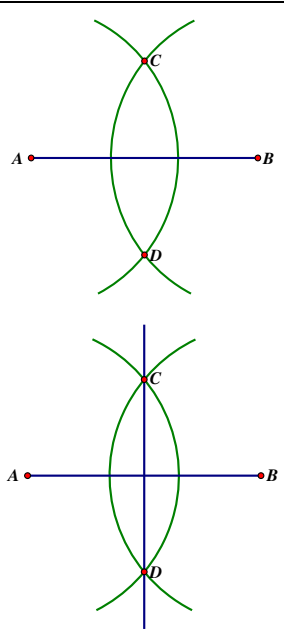

Fig. 1 The text about constructing the perpendicular bisector of a line segment 
Kirsch et al. have identified five categories of reading literacy: (1) retrieving information, (2) forming a general understanding, (3) developing an interpretation, (4) reflecting on and evaluating the contents of text, and (5) reflecting on and evaluating the forms of text. The first three categories focus on the meaning primarily derived from the text, whereas the remaining two categories emphasize comprehension of the meaning drawn from outside of the text. Furthermore, Kirsch et al. (2002) summarized the five categories as three categories of reading tasks: (1) retrieving information, (2) interpreting text, and (3) reflecting on content of and form of text. The three categories of reading tasks can be related to three main components of information processing: locating, integrating, and generating (Kirsch, 1995). Locating information means to literal matches between the question and the text, in which retrieving information from the text or focusing on independent parts of the text is required. Integrating information means to connect pieces of information from two or more locations (e.g. paragraphs, verbal and non-verbal information), in which making inferences based on text or focusing on relationships within the text is required. Generating information means to further process information, in which reflecting on the content and form of text on the basis of background or outside knowledge is required (Kirsch et al., 2002).

However, analyses only based on PISA's framework cannot significantly reveal the students' comprehension of geometric texts, especially when geometric figures are crucial parts of the texts. This situation requires including mathematical cognition in the dimension of reading literacy. Thus, we theoretically analyze the students' comprehension of the geometric construction text (see Fig. 1), based on Duval's (1995) apprehension of geometrical figures, and then coordinate the four kinds of apprehensions with the three categories of reading tasks. Before introducing the four kinds of figural apprehensions, we provide reasons to support our coordination of both PISA's assessment framework and Duval's cognitive framework into one construct. First, the development of PISA reading literacy assessment framework is rooted in cognition research which inevitably involves both learning product and process (Kirsch et al., 2002). Second, Kintsch (1998) stated that text comprehension can be viewed as a process of constructing multiple representations in relation to different depths of understanding. In line with Kintsch, mathematical text comprehension necessitates the recognition and inference of specific features embedded in mathematical discourse (e.g., terms, definitions, signs, contents, and structures) (OECD, 2004) for constructing comprehensive mental representations. Third, figures are crucial parts of geometric construction texts, and Duval (1995) has distinguished different kinds of figural apprehensions. Whereas comprehending geometric construction texts requires the interreference between construction steps and their corresponding figures, analyzing the possible cognitive apprehensions (Duval, 1995) underlying each category of reading tasks advances our understanding of reading comprehension regarding the genre of geometric construction texts.

Duval has distinguished four kinds of apprehension of geometrical figures as (1) perceptual apprehension: recognizing shapes and properties of figures in a plane or in space; (2) operative apprehension: transforming figures or reorganizing configurations; (3) sequential apprehension: constructing a figure or describing its construction in specific order; and (4) discursive apprehension: to explain or prove geometrical properties through natural speech or theoretical reasoning. 
With the construction of the perpendicular bisector of a given line segment as an example (see Fig. 1), to understand the geometric construction text requires not only perceptual apprehension of geometrical figures, which is helpful for recognizing the relationship between the line segment and the perpendicular bisector, but also sequential apprehension, which is helpful for making sense of the construction process. To further understand the construction process, students need discursive apprehension to distinguish the perceptual and sequential figures from the underlying reasons for the construction steps in the text based on accepted statements and deduction (Hanna, 1991; Mariotti, 2000). By operative apprehension, students can flexibly combine the subfigures constructed by a compass and a straightedge to examine the whole figure (the rhombus) and then to refocus on the given (the line segment) and finally the result (its perpendicular bisector). If students can coordinate these four kinds of apprehension of geometrical figures, they will more likely understand the logical relationship between geometrical figures and the underlying reasons why those construction steps can be used to accomplish the task.

After theoretically analyzing a process of comprehending the geometrical construction text, we coordinate the four kinds of apprehensions and the three categories of reading tasks. When geometric construction steps and their corresponding figures are read, perceptual apprehension is related to retrieving information mainly by focusing on a particular piece of information or a shape of a figure in the text. Sequential apprehension and operative apprehension - which require organization and transformation of figures based on technical constraints and mathematical properties (Duval, 1995) - are related to interpreting the relationships between several construction steps, as well as between figural and mathematical properties underlying a compass, e.g., a four-point figure and two of the four points constructed by a compass with a fixed opening. Discursive apprehension, which requires mathematical properties outside of the text for deducing how the object is constructed and the reason why the object can be derived from the construction steps, is related to reflecting on the contents and forms of the text.

In order to make the first dimension of the assessment framework more generalizable to other mathematical texts, we also relate the three categories of reading tasks to three clusters of task complexity, which is exploited to assess mathematical literacy in PISA (OECD, 2004). The three clusters, reproduction, connection, and reflection, can be analogous to retrieving information, interpreting, and reflecting. The reproduction cluster involves simple problems requiring only recall or routine skills through recognizing information; the connection cluster involves problems requiring interpretation or integration of information, and the reflection cluster involves problems requiring mathematical insight to generalize or validate (reasoning). Accordingly, we then propose three broader categories of reading comprehension of mathematical texts as retrieving or recognizing, interpreting or connecting, and reflecting or reasoning.

Besides reinterpreting the three categories of reading tasks by connecting them with mathematical literacy, the three categories are further distinguished on the basis of information sources for enhancing the content validity of the assessment framework. As for retrieving or recognizing, the information source comes from what is explicitly stated and illustrated in a text, for instance, words and figures. As for interpreting or connecting, the information source comes from both the text and the reader but is still based on text content mainly, for instance, the connection between words and figures, as well as the outcomes derived from construction actions. As for reflecting or reasoning, the information source comes from both the text and the reader but goes 
beyond the text content, for instance, why the geometric construction task can be accomplished by the construction steps. In view of information sources, the three categories of reading tasks can be contrasted as reading of, between, and beyond the text. In sum, the first dimension of our assessment framework is composed of these three categories and supported by reading literacy, apprehensions of geometrical figures, mathematical literacy as well as their connections. The detailed description of the three categories will be presented after the second dimension is identified.

In order to make our assessment framework more complete and valid, the second dimension considers the mathematical essence of geometric construction texts due to the effect of text content on reading comprehension. Thus, we try to identify the essential elements of geometric construction texts. Geometric construction texts are not only a special genre of mathematical texts but also different from geometric construction tasks. The elements necessary for geometric construction tasks can be identified as the required outcome, geometric properties, and basic geometric constructions (e.g., constructing a given segment or given angle). When thinking about how to construct a geometrical figure by compass and straightedge, students need to connect the required outcome with the relative geometric properties under the support and constraints in basic geometric construction, and then come up with the construction method. When comprehending the text of geometric construction, students need to distinguish the required outcome from the mathematical objects shown in the figure, to infer derived outcomes behind construction actions, and to reason why the required outcomes are obtained from the construction actions.

Just as premises/conditions, properties, and conclusions are viewed as the key elements of proof (Yang \& Lin, 2008), geometric construction texts also possess their own key elements. We identified in our study three key elements: (1) mathematical object which is acted on, (2) construction action with tools, and (3) outcome derived from action with tools after interviewing some school mathematics teachers and college professors. The exemplary opinions of four interviewees on geometric construction texts were provided as follows:

Mathematics teacher A: Some students (in general classrooms) have difficulties in knowing what can be derived from a construction action.

Mathematics teacher B: They follow the steps (action with tools) without thinking and do not know why we can follow these steps to construct what we want (outcome).

Mathematician C: A straightedge and a compass are simple and not complicated tools. They are used (action with tools) for activating our application of geometric knowledge. Students can learn how to "construct" and "produce" geometric knowledge (outcome).

Mathematician D: A geometric construction is constituted of construction rules (action with tools) and geometric knowledge underlying the constraints of a compass and a ruler.

The key elements mentioned above mainly focus on action with tools and outcome, that is, what can be constructed. However, what is built on each construction action is also important for understanding the reason underlying each step. Thus, we further distinguished object which is "acted on" from outcome. Object, which is acted on, is 
the given prior to one construction action; outcome, which is derived from action, emerges after a construction action. Moreover, outcome can become object in one construction step. For instance, the arcs drawn by step 1 in Fig. 1 are outcomes of the first action in step 1 and then become object of the second action in step 1, where points $\mathrm{C}$ and D are constructed. On the other hand, the sequence underlying step 1 which includes two actions can be considered as object and outcome, and each action in step 1 is accomplished by the use of a compass.

Besides, we can associate the three key elements with problem solving: the outcome from action is similar to the unknown, the mathematical object is viewed as the given, and the construction action with tools is considered as the method to connect the outcome with the object under certain conditions (Pólya, 1945). This association makes us more confident about using these three key elements in our study, but this does not imply that the reading comprehension of a geometric construction text is similar to problem solving in a geometric construction task.

To propose a two-dimensional framework for assessing reading comprehension of geometric construction texts, we cross-tabulated the three broader categories of reading tasks with the three key elements of geometric construction texts to create nine categories of reading comprehension of geometric construction texts (see Table 1). The meaning of each subcategory will be described and illustrated with examples in the next section. This framework can be adopted not only to measure students' learning outcomes but also to understand students' strength and weakness in reading comprehension of geometric construction texts.

\section{Applying the Framework to the Design of Assessment Questions}

For each category, we describe what is assumed to be assessed and then present exemplary questions which are used to assess students' reading comprehension of the

Table 1 A framework for assessing reading comprehension of geometric construction texts

\begin{tabular}{|c|c|c|c|}
\hline $\begin{array}{l}\text { Element } \\
\text { category }\end{array}$ & Objects acted upon & $\begin{array}{l}\text { Construction action with } \\
\text { tools }\end{array}$ & $\begin{array}{l}\text { Outcomes from action with } \\
\text { tools }\end{array}$ \\
\hline $\begin{array}{l}\text { Retrieving or } \\
\text { recognizing }\end{array}$ & $\begin{array}{l}\text { To know geometrical terms, } \\
\text { symbols, or visual figures } \\
\text { in text (Q1-1) }\end{array}$ & $\begin{array}{l}\text { To know the corresponding } \\
\text { relationship between } \\
\text { verbal and non-verbal text } \\
\text { of construction steps } \\
\text { (Q1-2) }\end{array}$ & $\begin{array}{l}\text { To know verbal or } \\
\text { non-verbal text that repre- } \\
\text { sents what is produced by } \\
\text { construction steps (Q1-3) }\end{array}$ \\
\hline $\begin{array}{l}\text { Interpreting or } \\
\text { connecting }\end{array}$ & $\begin{array}{l}\text { To interpret objects which } \\
\text { are acted upon or to } \\
\text { logically connect their } \\
\text { relationships (Q2-1) }\end{array}$ & $\begin{array}{l}\text { To interpret construction } \\
\text { steps or to connect } \\
\text { construction actions to } \\
\text { derive conditions (Q2-2) }\end{array}$ & $\begin{array}{l}\text { To interpret steps for } \\
\text { identifying the main } \\
\text { outcome to or to connect } \\
\text { the derived conditions } \\
\text { with the task goal (Q2-3) }\end{array}$ \\
\hline $\begin{array}{l}\text { Reflecting or } \\
\text { reasoning }\end{array}$ & $\begin{array}{l}\text { To reflect on the final } \\
\text { outcomes as mathematical } \\
\text { objects which are acted } \\
\text { upon and to reason one } \\
\text { step further (Q3-1) }\end{array}$ & $\begin{array}{l}\text { To reflect on the difference } \\
\text { and similarity between } \\
\text { the original steps and an } \\
\text { alternative construction } \\
\text { (Q3-2) }\end{array}$ & $\begin{array}{l}\text { To reflect on what is } \\
\text { constructed through an } \\
\text { alternative construction } \\
\text { and why the construction } \\
\text { can construct it (Q3-3) }\end{array}$ \\
\hline
\end{tabular}


following geometric construction text as shown in Fig. 2. The text can be read and understood to some extent by students who have learned how to construct the perpendicular bisector of a given line segment and the bisector of a given angle.

\section{Category of Retrieving or Recognizing}

Referring to PISA's definition of reading and mathematical literacy, we defined in our study the category of retrieving or recognizing information as the identification of geometrical symbols, perceptual features of figures, and the relationship between verbal text (language and symbols) and non-verbal text (drawings and figures), which can correspond to Duval's (1995) non-discursive (language and drawings) and discursive register (symbols and figures). With respect to the elements of mathematical objects which are acted upon, readers are expected to recognize geometrical terms, mathematical symbols, or geometrical figures used in one specific step of construction (e.g., Q1-1 in Fig. 3). With respect to the elements of construction action, readers are expected to retrieve the corresponding relationship between verbal text and non-verbal text (figures) in each construction action (e.g., Q1-2 in Fig. 3). With respect to the elements of outcome from action, readers are expected to retrieve or recognize verbal text and non-verbal text (figures) which are produced by one construction action (e.g., Q1-3 in Fig. 3).

In Q1-1 in Fig. 3, the students are asked to recognize the meaning of the symbol $\overline{A B}$ in the geometric construction context. The meaning of both line segment and its length is required for constructing the perpendicular bisector. In Q1-2 in Fig. 3, the students are asked to recognize the two loci for the construction in step 1 in Fig. 1. In Q1-3 in Fig. 3, the students are asked to distinguish the outcome from the construction action by

Given $\overline{A B}$, construct a $45^{\circ}$ angle on the given segment.
Construct the line, $\overleftrightarrow{P Q}$,
as the perpendicular bisector of $\overline{A B}$.
[Step 2] and $\overline{A B}$ intersect at $\mathrm{O}$.
Construct the line, $\overleftrightarrow{\mathrm{OC}}$,
as the bisector of $\angle \mathrm{BOP}$.

Fig. 2 The text about constructing a $45^{\circ}$ angle on the given segment 
Q 1-1. What does $\overline{A B}$ mean in Step 1? (multiple answers)

$\square$ The length bet weentwo points A and B.

$\square$ The segment from point A to point B.

$\square$ The length of the line passing through two points A and B.

$\square$ The line passing through two points A and B.

Q 1-2. Referring to Step 1 in Figure 1, which two loci in the following figure are required for constructing the perpendicular bisector of $\overline{A B}$ ? (Circle them or Mark them with full lines)

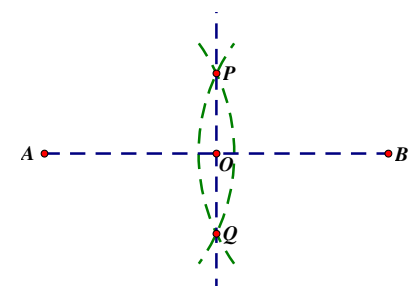

Q 1-3. Referring to Step 1 in Figure 1, which locus in the following figure is the perpendicular bisector of $\overline{A B}$ ? (Circle it or Mark it with a full line )

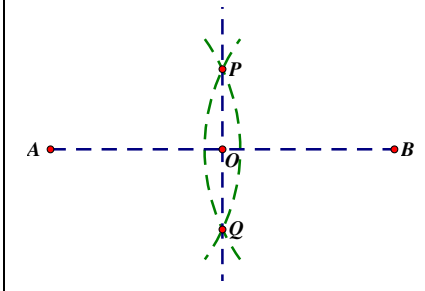

Fig. 3 Assessment questions with respect to retrieving or recognizing

retrieving their preknowledge of the perpendicular bisector of a given segment. Retrieving or recognizing information in text is necessary to answer these questions.

\section{Category of Interpreting or Connecting}

We define this category as interpreting or connecting information within or between verbal and non-verbal text. With respect to the elements of mathematical objects which are acted upon, readers are expected to interpret mathematical objects which are acted upon or connect their logical relationships, for example, Q2-1 in Fig. 4 asks the students to order the objects which are built on to construct a $45^{\circ}$ angle. With respect to the elements of construction action, readers are expected not only to interpret steps 1 and 2 but also to connect each action constructed by using a compass with its 
Q 2-1. Re-arrange the three objects based on the appearance order by referring to
Step 2 in Figure 2.
(1) Arc RS,
(2) $\overline{O C}$,
(3) two arcs at point $\mathrm{C}$.

Q 2-2. Referring to Steps 1 and 2 in Figure 2, which of the following conditions can be derived? (Multiple answers)
$\overline{P A}=\overline{P Q}$
$\overline{Q A}=\overline{Q B}$
$\angle B O P=90^{\circ}$
$\overline{O A}=\overline{O R}$
$\overline{C R}=\overline{C B}$
$\angle P O C=\angle B O C$
$\overline{O P}=\overline{O Q}$

Q 2-3. Of the above answers you have selected, which answer is the main purpose of this task? (Single answer)

Fig. 4 Assessment questions with respect to interpreting or connecting

underlying property (e.g., Q2-2 in Fig. 4). With respect to the elements of outcome from action, readers are expected to interpret the derived conditions by connecting the goal of the task with the bisector of a $90^{\circ}$ angle (e.g., Q2-3 in Fig. 4).

In Q2-1 in Fig. 4, the students are asked to logically connect objects which are sequentially constructed and then built upon in step 2 in Fig. 2. In Q2-2, the students are asked to connect construction actions to derive conditions. For example, " $\overline{\mathrm{QA}}=\overline{\mathrm{QB}}, \angle \mathrm{BOP}=90^{\circ}$ and $\overline{\mathrm{OP}}=\overline{\mathrm{OQ}}$ " can be derived from step 1 . In Q2-3, the students are asked to identify the main outcome according to the purpose of this task. Interpreting or connecting information within and between verbal and non-verbal text is necessary to answer these questions.

\section{Category of Reflecting or Reasoning}

We define this category as reflecting on what is constructed or reasoning why the construction is valid. With respect to the elements of mathematical objects which are acted upon, readers are expected to reflect on the final outcome as mathematical objects (e.g., Q3-1 in Fig. 5). With respect to the elements of construction action, readers are expected to reflect on the original steps for identifying the differences and similarities between this construction task and an alternative construction task (e.g., Q3-2 in Fig. 5). With respect to the elements of outcome from action, readers are expected to reflect on what is constructed in an alternative construction task and why it can be constructed (e.g., Q3-3 in Fig. 5).

In Q3-1 in Fig. 5, the students are asked to reflect on the original construction task for constructing a $67.5^{\circ}$ angle. In Q3-2, the students are asked to reflect on the original figure and the alternative construction task. If students can analogize the objects $\overline{\mathrm{OA}}$ $=\overline{\mathrm{OB}}$ and line $\mathrm{PQ}$, in the original construction to the objects $\overline{\mathrm{OP}}=\overline{\mathrm{OQ}}$ and line $\mathrm{OS}$ in the alternative construction task, they will more likely point out the main difference between the two construction steps. In Q3-3, the students are asked to reflect on the goal of the alternative construction task, that is, what is constructed, and to prove that the construction task steps can be followed to construct the geometrical figure required. In proving this, the students need to identify the proof goals, for example, why line OR is perpendicular to $\overline{\mathrm{AB}}$ and why $\angle \mathrm{COT}$ is a $45^{\circ}$ angle. Reflecting or reasoning beyond text is necessary to answer these questions. 
Q 3-1. Based on the outcome of Steps 1 and 2 in Figure 2, how can you construct a $67.5^{\circ}$ angle by one more step? Briefly write down the steps and draw it on the following figure.

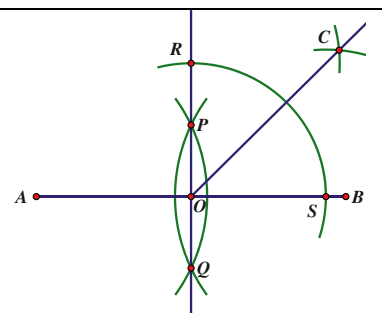

Q 3-2. Chun-Jiao adopts an alternative construction step to accomplish the task.

What is the main difference between the original construction and the following three-step construction?

[Step 1]

Select one point $\mathrm{O}$ from $\overline{A B}$ as shown in the following figure.

[Step 2]

Construct one line which is the perpendicular of $\overline{A B}$

and passes through the point $\mathrm{O}$. Label three points $\mathrm{P}, \mathrm{Q}$, and R.

[Step 3]

Construct the bisector of $\angle \mathrm{BOR}$. Label three points $\mathrm{S}, \mathrm{T}, \mathrm{C}$.

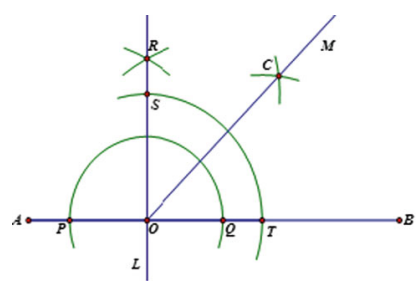

Q 3-3. What is the construction task that can be inferred from the above three-step construction? And prove why it can be constructed by following these construction steps.

Fig. 5 Assessment questions with respect to reflecting or reasoning

\section{Validating the Instrument for Assessing Reading Comprehension of Geometric Construction Texts}

We have applied the above comprehensive conceptual framework to develop an assessment instrument and to meet content validity requirements because this framework serves as a theoretical basis for formulating operational definitions of reading comprehension of geometric construction texts. The concept of validity pertains to the degree to which an instrument assesses what it claims to assess from a perspective of 
internal validity (see Lissitz \& Samuelsen, 2007). To improve the validity and minimize measurement errors in this study, the questions were evaluated by two mathematics education researchers and tested in a pilot classroom. From the mathematics education researchers' feedback and students' responses, we revised the wording in some items. For instance, the term “中- - " (the Chinese term literally means "middle-vertical-line”) was revised as “垂直- 分-” (the Chinese term literally means “perpendicular-bisectline") to make this term semantically clearer. In order to clearly identify construction actions which require students to infer some derived properties, the steps were labeled in the text as shown in Fig. 2 and referred to in questions as shown in Figs. 3, 4, and 5. In Q3-1, the information " $67.5^{\circ}=45^{\circ}+\left(\frac{45}{2}\right)^{\circ}$ " was replaced by $67.5^{\circ}$ to encourage students to reflect upon how this information is obtained. In Q3-3, "to explain why" was revised as "to prove why" in order to facilitate students' formal argumentation.

In addition, we conducted a questionnaire survey to validate the instrument for assessing reading comprehension of geometric construction texts of constructing a $45^{\circ}$ angle as shown in Fig. 2. The number of questions with respect to each element and each reading category is displayed in Table 2. The instrument was administered to 219 junior high school students in grade 9 who had learned geometric constructions in grade 8 . All the participants came from 18 classes at the same public junior high school in Taoyuan County in Taiwan. When students enrolled in this school, they were randomly assigned to classes so that the students' mean scores on an intelligence test were not significantly different from each other in each class. When one third of the students in those classes answered the question in the instrument, the others answered questions in the other two instruments for another project on reading mathematics. Of the 219 students, 197 students provided analyzable data.

For open questions, partial credit was assigned if a response is not fully correct. For multiple choices with single correct answer, responses were scored " 1 " for a correct answer and " 0 " for an incorrect answer. For multiple choices with multiple correct answers, partial credit was assigned depending on the ratio of correct responses to the number of choices. For instance, if there were five choices that are correct answers in one question and only two choices were correctly responded to, then a point of two

Table 2 Characteristics of questions and students' average scores

\begin{tabular}{lllll}
\hline Characteristics & & $\begin{array}{l}\text { Number of } \\
\text { questions }\end{array}$ & $\begin{array}{l}\text { Average discrimination } \\
\text { index }\end{array}$ & $\begin{array}{l}\text { Students' average } \\
\text { scores (range 0-1) }\end{array}$ \\
\hline Rategory & Element & & & \\
\hline Retrieving/recognizing & Object & 1 & 0.327 & 0.614 \\
& Action & 1 & 0.434 & 0.858 \\
& Outcome & 1 & 0.755 & 0.635 \\
Interpreting/connecting & Object & 1 & 0.434 & 0.863 \\
& Action & 3 & 0.493 & 0.781 \\
& Outcome & 3 & 0.563 & 0.646 \\
Reflecting/reasoning & Object & 2 & 0.807 & 0.463 \\
& Action & 1 & 0.858 & 0.418 \\
& Outcome & 3 & 0.651 & 0.410 \\
\hline
\end{tabular}


fifths was assigned. The Cronbach's alpha reliability coefficient of this instrument was 0.89 for the participating students of grade 9. Average item discrimination index values and students' average scores for each category are displayed in Table 2. The item discrimination - that measures the differences between the average scores of students in the upper $27 \%$ group $(\mathrm{H})$ and those of the lower $27 \%$ group (L) - was calculated using the formula $\mathrm{d}=\mathrm{H}-\mathrm{D}$. Except for the first question Q1-1, all of the item discrimination index values were higher than 0.4 , which can be regarded as indicating good item discrimination power.

Table 2 shows that the highest two average scores of students' performance appear in the tasks of retrieving/recognizing construction action and of interpreting/connecting object, respectively. The result indicates that more than $85 \%$ of students can know the relationship between verbal and non-verbal text of construction steps (Q1-2) and can interpret objects which are acted upon or to logically connect their relationships (Q2-1) in the two-step geometric construction text. The result also implies that retrieving/ recognizing tasks is not absolutely easier for students than interpreting/connecting tasks. For instance, it is cognitively demanding for students to recognize the meaning of geometric symbols. We find that about $40 \%$ of students cannot correctly identify the meanings of the symbol, $\overline{\mathrm{AB}}$, as the segment from point $\mathrm{A}$ to point $\mathrm{B}$ as well as the length of the segment. They misinterpret the symbol, $\overline{\mathrm{AB}}$ as the length of the line passing through two points $\mathrm{A}$ and $\mathrm{B}$.

Moreover, Table 2 shows that all of the lowest three average scores appear in reflecting/reasoning on object, action, and outcome. The result implies that almost half of the students cannot view the final outcomes as mathematical objects (Q3-3) and cannot identify the difference and similarities among the original construction steps as well as the alternative construction steps (Q3-2). As for Q3-1, students failing to know how to construct a $67.5^{\circ}$ angle by one more step may be resulted from not attending to view $67.5^{\circ}$ as $45^{\circ}+\left(\frac{45}{2}\right)^{\circ}$, i.e., no attention to recognizing relationships (Mason, 2003). Table 3 displays the distribution of students' scores on reading comprehension of geometric construction texts. Although the students had learned how to construct a $45^{\circ}$ angle, almost half of them did not obtain scores higher than two thirds of the total scores. It implies that students who are able to do a geometric construction task are not necessarily able to comprehend a geometric construction text of the task.

\section{Discussion}

\section{Specific and Generalizable Nature of this Assessment Framework}

In this article, we consider both categories of reading mathematical texts and elements of geometric construction texts to develop a two-dimensional framework for assessing reading comprehension of geometric construction texts in our study. However, the

Table 3 Distribution of students' scores

\begin{tabular}{llll}
\hline Students' scores & $0-3$ & $3-6$ & $6-9$ \\
\hline Percentage & $13.20 \%$ & $38.57 \%$ & $48.23 \%$ \\
\hline
\end{tabular}


generalizability of the two-dimensional framework is not limited to geometric construction texts. The three categories of recognizing/retrieving, interpreting/connecting, and reflecting/reasoning can be applied to comprehending mathematical texts because they are also corresponding to three levels of mathematical literacy in PISA.

As for the first two categories, recognizing/retrieving and interpreting/connecting are similar to but still different from the original meanings in PISA, because recognizing/ retrieving and interpreting/connecting mathematical texts heavily depend on the readers' prior knowledge of a text, which influences their reading comprehension of the text (Johnston, 1984). As for the third category, reflecting/reasoning differs from its original meaning in PISA regarding what students are required to reflect on. Reading mathematical texts requires readers to reflect on what is derived or validated, the underlying mathematical structure, application, and extensions (Inglis \& Alcock, 2012; Mejia-Ramos et al., 2012; Shepherd \& van de Sande, 2014). Drawing on the specific and generalizable natures of the two-dimensional framework, our approachto identify key elements of geometric construction texts and then to cross-tabulate with the above three categories of reading mathematical texts - can be applied as a general framework for assessing students' comprehension of mathematical texts.

\section{Implications of this Assessment Framework}

Our nine-category assessment framework has responded to the need for better comprehension tests (Kintsch, 2012) and gives a concrete picture of comprehending geometric construction texts from the basic level of recognizing/retrieving, the transformative level of interpreting/connecting to the encapsulated level of reflecting/ reasoning through the three elements of geometric construction texts.

To address the gap between visualization and geometrical reasoning in constructing geometrical figures with a compass and straightedge (Mariotti, 1995), we propose reading-to-learn activities where part of visualization is supported by figures in the texts, and geometric properties applied to the construction can be inferred from the constructions steps in the texts. In particular, students' need for proof can be facilitated by the question to identify what should be proved, and proving follows. The need for proof and proving offers students opportunities to clarify their understanding through writing (Stempien \& Borasi, 1985). The assessment instrument for reading comprehension of geometric construction texts provides the learning opportunity to organize and connect geometrical knowledge and figures presented in the texts with what learners have already known, to distinguish spatial-graphical drawings from geometrical figures, and to conjecture the properties underlying construction steps.

Pandiscio (2002) supposed that geometric construction tasks promote students' problem solving through the use of reasoning, and that these tasks illustrate how geometric constructions can broaden and deepen students' mathematical perspective through requiring them to construct geometrical figures by using different ways and tools. Herein, we propose an alternative but complementary approach to teaching geometric constructions with the perspective of reading to learn mathematics (Borasi \& Siegel, 1990). Our assessment framework can support teachers to design reading instruction about making meanings from geometric construction texts. Like what Weinberg and Wiesner (2011) suggested - “instructors can use reading questions to encourage students to wrestle actively with the mathematical ideas presented in the 
textbook and to construct new meaning from their own ideas" (p. 61)—our assessment framework and exemplary questions could serve as a basis for designing comprehension questions.

We believe that this study not only provides an assessment framework illustrating the meaning of reading comprehension of geometric construction texts but also integrates reading literacy and mathematical texts for making content area reading instruction more accessible for mathematics teachers.

Acknowledgments We thank the reviewers for their helpful suggestions. This study is supported by the Ministry of Science and Technology of Taiwan (MOST 102-2511-S-003-022-MY3).

Open Access This article is distributed under the terms of the Creative Commons Attribution 4.0 International License (http://creativecommons.org/licenses/by/4.0/), which permits unrestricted use, distribution, and reproduction in any medium, provided you give appropriate credit to the original author(s) and the source, provide a link to the Creative Commons license, and indicate if changes were made.

\section{References}

Australian Curriculum, Assessment and Reporting Authority (2012). The Australian Curriculum: Mathematics (V3.0). Retrieved from http://www.australiancurriculum.edu.au/Australian\%20Curriculum. pdf?Type $=0 \& \mathrm{~s}=\mathrm{M} \& \mathrm{e}=$ ScopeAndSequence

Borasi, R., \& Siegel, M. (1989). Reading to learn mathematics: A new synthesis of the traditional basics. Paper presented at the Annual Meeting of the American Educational Research Association, San Francisco, CA.

Borasi, R. \& Siegel, M. (1990). Reading to learn mathematics: New connections, new questions, new challenges. For the Learning of Mathematics, 10(3), 9-16.

Common Core State Standards Initiative (2012). Common core state standards initiative: Preparing America's students for college and career. Retrieved from http://www.corestandards.org/

Conradie, J. \& Frith, J. (2000). Comprehension tests in mathematics. Educational Studies in Mathematics, 42(3), 225-235.

Department for Education (2013). National curriculum in England: Mathematics programmes of study. Retrieved from https://www.gov.uk/government/publications/national-curriculum-in-englandmathematics-programmes-of-study/national-curriculum-in-england-mathematics-programmes-of-study

Duval, R. (1995). Geometrical pictures: Kinds of representation and specific processings. In R. Sutherland \& F. Mason (Eds.), Exploiting mental imagery with computers in mathematics education (pp. 142-157). Berlin, Germany: Springer.

Duval, R. (2006). A cognitive analysis of problems of comprehension in a learning of mathematics. Educational Studies in Mathematics, 61(1), 103-131.

Fang, Z. \& Schleppegrell, M. J. (2010). Disciplinary literacies across content areas: Supporting secondary reading through functional language analysis. Journal of Adolescent \& Adult Literacy, 53(7), 587-597.

Hanna, G. (1991). Mathematical proof. In D. Tall (Ed.), Advanced mathematical thinking (pp. 54-61). Dordrecht, The Netherlands: Kluwer.

Hölzl, R. (1995). Between drawing and figure. In R. Sutherland \& J. Mason (Eds.), Exploiting mental imagery with computers in mathematics education (pp. 117-124). Berlin, Germany: Springer.

Inglis, M. \& Alcock, L. (2012). Expert and novice approaches to reading mathematical proofs. Journal for Research in Mathematics Education, 43(4), 358-390.

Johnston, P. (1984). Prior knowledge and reading comprehension test bias. Reading Research Quarterly, 19(2), 219-239.

Kintsch, W. (1998). Comprehension: a paradigm for cognition. New York, NY: Cambridge University Press.

Kintsch, W. (2012). Psychological models of reading comprehension and their implications for assessment. In J. P. Sabatini, E. R. Albro \& T. O’Reilly (Eds.), Measuring up: Advances in how we assess reading ability (pp. 21-38). Plymouth, UK: Rowman \& Littlefield. 
Kirsch, I. (1995). Literacy performance on three scales: Definitions and results. In OECD \& Statistics Canada (Ed.), Literacy, economy and society. Results of the first international adult literacy survey (pp. 27-53). Paris, France: OECD.

Kirsch, I., de Jong, J., LaFontaine, D., McQueen, J., Mendelovits, J. \& Monseur, C. (2002). PISA reading for change: Performance and engagement across countries. Paris, France: Organisation for Economic Cooperation and Development.

Laborde, C. (1998). Relationships between the spatial and theoretical in geometry: The role of computer dynamic representations in problem solving. In D. Tinsley \& D. Johnson (Eds.), Information and communications technologies in school mathematics (pp. 183-194). New York, NY: Springer.

Lissitz, R. W. \& Samuelsen, K. (2007). A suggested change in terminology and emphasis regarding validity and education. Educational Researcher, 36(8), 437-448.

Mariotti, M. A. (1995). Images and concepts in geometrical reasoning. In R. Sutherland \& F. Mason (Eds.), Exploiting mental imagery with computers in mathematics education (pp. 97-116). Berlin, Germany: Springer.

Mariotti, M. A. (2000). Introduction to proof: the mediation of a dynamic software environment. Educational Studies in Mathematics, 44(1-2), 25-53.

Mason, J. (2003). On the structure of attention in the learning of mathematics. Australian Mathematics Teacher, 59(4), 17-25.

Mejia-Ramos, J. P., Fuller, E., Weber, K., Rhoads, K. \& Samkoff, A. (2012). An assessment model for proof comprehension in undergraduate mathematics. Educational Studies in Mathematics, 79(1), 3-18.

Organisation for Economic Co-operation and Development (OECD). (2004). The PISA 2003 assessment framework: mathematics, reading, science and problem solving knowledge and skills. Paris, France: Author.

Pandiscio, E. A. (2002). Alternative geometric constructions: Promoting mathematical reasoning. The Mathematics Teacher, 95(1), 32-36.

Pólya, G. (1945). How to solve it. Princeton, NJ: Princeton University.

Robertson, J. M. (1986). Geometric constructions using hinged mirrors. The Mathematics Teacher, 79(5), $380-386$.

Sabatini, J., Albro, E. \& O'Reilly, T. (2012). Measuring up: Advances in how we assess reading ability. Lanham, MD: R\&L Education.

Sanders, C. V. (1998). Geometric constructions: Visualizing and understanding geometry. The Mathematics Teacher, 91(7), 554-556.

Schoenfeld, A. (1986). On having and using geometric knowledge. In J. Hiebert (Ed.), Conceptual and procedural knowledge: The case of mathematics (pp. 225-264). Hillsdale, NJ: Erlbaum.

Selden, A. \& Shepherd, M. D. (2013). The Importance of and the need for research on how students read and use their mathematics textbook (technical report no. 3). Cookeville, TN: Tenessee Technological University.

Shepherd, M. D., Selden, A. \& Selden, J. (2012). University students' reading of their first-year mathematics textbooks. Mathematical Thinking and Learning, 14(3), 226-256.

Shepherd, M. D. \& van de Sande, C. C. (2014). Reading mathematics for understanding-From novice to expert. The Journal of Mathematical Behavior, 35, 74-86.

Stempien, M. \& Borasi, R. (1985). Students' writing in mathematics: Some ideas and experiences. For the Learning of Mathematics, 5(3), 14-17.

Weinberg, A. \& Wiesner, E. (2011). Understanding mathematics textbooks through reader-oriented theory. Educational Studies in Mathematics, 76(1), 49-63.

Yang, K.L. (2015). Study on junior high school students' reading to learn geometry (Report No. MOST. 1022511-S-003-022-MY3). Unpublished Technical Report. Taipei, Taiwan: Ministry of Science and Technology.

Yang, K. L. \& Lin, F. L. (2008). A model of reading comprehension of geometry proof. Educational Studies in Mathematics, 67(1), 59-76. 